\title{
Biophysical and in silico characterization of NrtA: A protein- based host for aqueous nitrate and nitrite recognition
}

\author{
Ke Ji ${ }^{a}$, Kiheon Baek ${ }^{a}$, Weicheng Peng ${ }^{\mathrm{ab}}$, Kevin A. Alberto ${ }^{\mathrm{a}}$, Hedieh Torabifard ${ }^{\mathrm{a}}$, Steven O. \\ Nielsen ${ }^{\star a}$, and Sheel C. Dodani ${ }^{* a}$

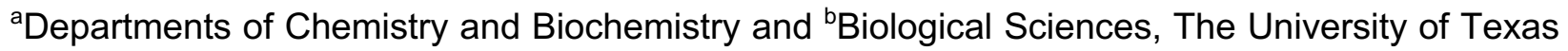 \\ at Dallas, Richardson, TX 75080 \\ *sheel.dodani@utdallas.edu, steven.nielsen@utdallas.edu
}

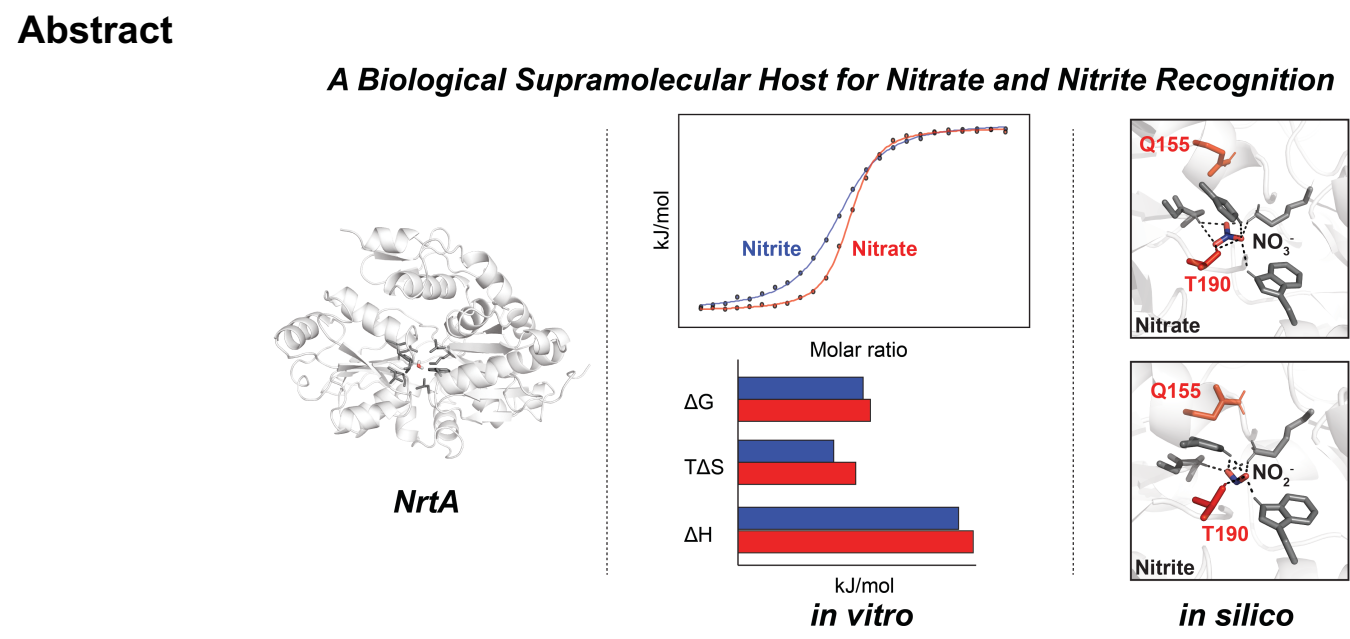

Nitrate and nitrite are key components of the global nitrogen cycle. As such, Nature has evolved proteins as biological supramolecular hosts for the recognition, translocation, and transformation of both nitrate and nitrite. To understand the supramolecular principles that govern these anionprotein interactions, here, we employ a hybrid biophysical and in silico approach to characterize the thermodynamic properties and protein dynamics of NrtA from the cyanobacterium Synechocystis sp. PCC 6803 for the recognition of nitrate and nitrite.

\section{Main Text}

Nitrate and nitrite are essential inorganic anions connecting all forms of life and their environments. ${ }^{1}$ In bacterial and plant cells, nitrate is a substrate for assimilatory and aerobic/anaerobic respiration pathways via the interconversion of nitrate, nitrite, and ammonium; in animal cells, nitrate and nitrite are thought to be storage reservoirs for nitric oxide homeostasis affording therapeutic potential. ${ }^{2-6}$ Their natural distributions can be perturbed through anthropogenic means, particularly by excessive crop fertilization leading to water pollution and eutrophication. ${ }^{7,8}$ Across all these processes, cells must dynamically recognize, translocate, and transform these anions to maintain homeostasis.

Along these lines, Nature has evolved proteins as biological supramolecular hosts to form thermodynamically stable complexes with nitrate and/or nitrite in water ${ }^{9-17}$ Representative crystal 
structures for a subset of these proteins indicate that this recognition is achieved by combining the hydrophobic effect with cooperative hydrogen bonding, ion-pairing, and van der Waals interactions. These supramolecular designs are tailored to the properties of the anions, including size (nitrate: 179 pm; nitrite: 192 pm), shape (trigonal planar vs. bent), charge (monoanionic), basicity (nitrate < nitrite), and hydration enthalpy (nitrate: $310 \mathrm{~kJ} / \mathrm{mol}$; nitrite: $410 \mathrm{~kJ} / \mathrm{mol}$ ). ${ }^{18}$ Indeed, protein-based hosts have served as the inspiration to design synthetic supramolecular hosts for nitrate and nitrite recognition, for which there are few aqueous examples. ${ }^{19-23}$ To better inform this design, we are actively studying interactions between nitrate/nitrite and prokaryotic protein-based hosts as part of a larger program aimed at decoding the supramolecular principles of biological anion recognition. ${ }^{24-26}$ Here, we have developed a hybrid workflow using isothermal titration calorimetry (ITC) and molecular dynamics (MD) simulations to dissect the thermodynamic contributions and protein motions of NrtA from the cyanobacterium Synechocystis sp. PCC 6803 upon nitrate and nitrite binding. ${ }^{9}$

NrtA is a periplasmic solute binding lipoprotein of the $A B C$ transport system NrtABCD that sequesters nitrate and nitrite from the environment. ${ }^{27,28}$ It delivers nitrate and nitrite from the periplasm to NrtB, an inner membrane spanning protein that translocates the anions into the cytosol. The latter transport process is coupled to the hydrolysis of ATP through the action of the membrane associated partners NrtC and NrtD. Based on the crystal structure of the nitrate-bound form of NrtA from Synechocystis sp. PCC 6803, NrtA is composed of two globular domains that create a cleft for the nitrate binding site. ${ }^{9}$ Inside the binding pocket, K269 serves as an anchor for nitrate binding and Q155, H196, and G240 provide additional hydrogen bonding interactions. These polar interactions are further supported by hydrophobic residues, L71, W102, L124, P222, and V239, that likely enable the dehydration of the anion (Fig. 1). Beyond these structural insights, the binding of nitrate and nitrite to NrtA from Synechocystis sp. PCC 6803 has not been further characterized until this study. However, the nitrate and nitrite dissociation constants $\left(K_{d}\right)$ have been determined for two different homologues using equilibrium dialysis coupled to mass spectrometry or colorimetric outputs (Table S1). For NrtA from Synechococcus elongatus, the affinities for nitrate $\left(K_{d}=0.32 \mu \mathrm{M}\right)$ and nitrite $\left(K_{d}=0.34 \mu \mathrm{M}\right)$ at $30^{\circ} \mathrm{C}$ were comparable; however, for NrtA from Phormidium laminosum the affinity for nitrate $\left(K_{d}=2.0 \pm 0.3 \mu \mathrm{M}\right)$ was greater than that for nitrite $\left(K_{d}=3.8 \pm 0.4 \mu \mathrm{M}\right)$ at $45{ }^{\circ} \mathrm{C} .{ }^{29,30}$

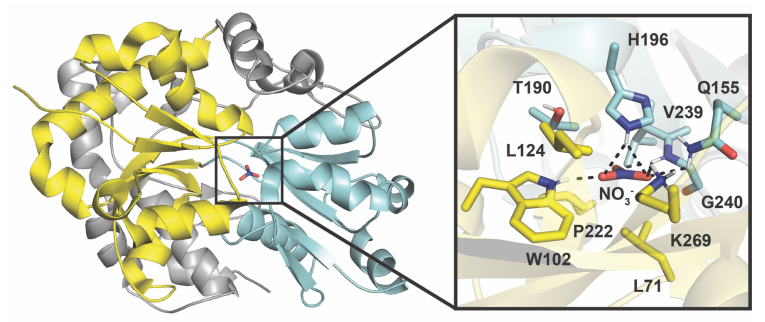

Fig. 1 X-ray crystal structure of nitrate-bound NrtA (residues 57-441, PDB ID: 2G29) from Synechocystis sp. PCC 6803. The two globular domains are highlighted in yellow and cyan (left). Residues within $4 \AA$ of the nitrate ion are shown as sticks with all oxygen atoms in red, nitrogen atoms in blue, and hydrogen atoms in white (right). All known hydrogen bonding or electrostatic interactions with nitrate are shown with dashed lines. Each residue is labeled with the single letter amino acid abbreviation and corresponding sequence number. 
Given the similarities in the binding pocket of these homologues based on sequence alignments, we first used high-throughput differential scanning fluorimetry to determine if nitrate and nitrite could stabilize NrtA from Synechocystis sp. PCC 6803 upon binding (Figs. S1-S5; Table S2). ${ }^{31,32}$ The average unfolding temperature $\left(T_{m}\right)$ of apo NrtA is $75.19 \pm 0.18{ }^{\circ} \mathrm{C}$ in $20 \mathrm{mM} \mathrm{HEPES}$ at pH 7.5 with $100 \mathrm{mM} \mathrm{NaCl}$. Titration with both nitrate and nitrite increases the $T_{m}$ in a dose-dependent manner with saturation of binding, indicating the formation of a stable complex with NrtA. With respect to apo NrtA, the average $\Delta \mathrm{T}_{\mathrm{m}}$ increases by $3.72 \pm 0.45^{\circ} \mathrm{C}$ up to $15.74 \pm 0.20{ }^{\circ} \mathrm{C}$ with 100 $\mu \mathrm{M}$ and $100 \mathrm{mM}$ sodium nitrate, respectively. However, at the same concentrations of sodium nitrite, the average $\Delta \mathrm{T}_{\mathrm{m}}$ is smaller, $1.04 \pm 0.65{ }^{\circ} \mathrm{C}$ and $12.42 \pm 0.20{ }^{\circ} \mathrm{C}$, suggesting a weaker complex. Similar results are not observed with sodium gluconate $\left(\Delta \mathrm{T}_{\mathrm{m}}<1{ }^{\circ} \mathrm{C}\right)$, removing the possibility of ionic strength effects. To further support that nitrate and nitrite bind in the same pocket, we introduced the K269A mutation, which has been speculated to contribute to binding in a homologous bicarbonate binding protein. ${ }^{9,33}$ Under identical conditions, the $\mathrm{T}_{\mathrm{m}}$ of apo NrtA $\mathrm{K} 269 \mathrm{~A}$ is $78.34 \pm 0.50^{\circ} \mathrm{C}$, and no concentration-dependent stabilization occurs with nitrate, nitrite, or gluconate $\left(\Delta \mathrm{T}_{\mathrm{m}}<1^{\circ} \mathrm{C}\right)$, thus confirming that NrtA can bind both nitrate and nitrite using the same binding pocket (Fig. S6; Table S3).

Building off this qualitative measure of recognition, we next employed ITC to quantify the binding affinities $\left(K_{d}\right)$ and associated enthalpy and entropy contributions for both nitrate and nitrite. ${ }^{34}$ At $20{ }^{\circ} \mathrm{C}$ in $20 \mathrm{mM}$ HEPES at $\mathrm{pH} 7.7$ with $50 \mathrm{mM} \mathrm{NaCl}$, both ions undergo an exothermic binding reaction with a $1: 1$ stoichiometry (Figs. 2, S7; Tables 1, S4). Moreover, we verified that there is no proton uptake or release coupled to anion binding under these conditions by testing binding in buffers (e.g., HEPES, phosphate, Tris) with different ionization enthalpies (Figs. S8-S10; Table S4). ${ }^{35}$

Table 1. Thermodynamic parameters for nitrate and nitrite binding to NrtA at different temperatures. All experiments were carried out in $20 \mathrm{mM}$ HEPES with $50 \mathrm{mM} \mathrm{NaCl}$ at $\mathrm{pH}$ 7.7. The average of two protein preparations, each measured in duplicate with the standard deviation is shown.

\begin{tabular}{cccccccc}
\hline Anion & $\begin{array}{c}\text { Temperature } \\
\left({ }^{\circ} \mathbf{C}\right)\end{array}$ & $\begin{array}{c}\boldsymbol{K}_{\mathrm{d}} \\
(\mathbf{n M})\end{array}$ & $\begin{array}{c}\mathbf{\Delta} \mathbf{H} \\
\left(\mathbf{k J} \cdot \mathbf{m o l}^{-1}\right)\end{array}$ & $\begin{array}{c}\mathbf{T} \mathbf{\Delta} \\
\left(\mathbf{k J} \cdot \mathbf{m o l}^{-1}\right)\end{array}$ & $\begin{array}{c}\Delta \mathbf{G} \\
\left(\mathbf{k J} \cdot \mathbf{m o l}^{-1}\right)\end{array}$ & $\begin{array}{c}\Delta \mathbf{C}_{\mathbf{p}} \\
\left(\mathbf{J} \cdot \mathbf{m o l}^{-1} \mathbf{K}^{-1}\right)\end{array}$ & $\mathbf{n}$ \\
\hline \multirow{3}{*}{ Nitrate } & 10 & $48 \pm 8$ & $-65.0 \pm 0.7$ & $-25.3 \pm 0.5$ & $-39.7 \pm 0.4$ & & $1.02 \pm 0.02$ \\
& 20 & $57 \pm 23$ & $-68.0 \pm 1.2$ & $-27.2 \pm 1.7$ & $-40.7 \pm 1.0$ & & $1.06 \pm 0.02$ \\
& 30 & $88 \pm 9$ & $-70.3 \pm 2.5$ & $-29.3 \pm 2.3$ & $-41.0 \pm 0.3$ & $-209 \pm 45$ & $1.06 \pm 0.04$ \\
& 37 & $115 \pm 8$ & $-70.5 \pm 1.2$ & $-29.3 \pm 1.2$ & $-41.2 \pm 0.2$ & & $1.06 \pm 0.05$ \\
\hline \multirow{3}{*}{ Nitrite } & 10 & $187 \pm 16$ & $-56.3 \pm 0.5$ & $-19.8 \pm 0.8$ & $-36.5 \pm 0.3$ & & $1.05 \pm 0.06$ \\
& 20 & $230 \pm 33$ & $-60.6 \pm 1.1$ & $-23.4 \pm 1.5$ & $-37.3 \pm 0.4$ & $-463 \pm 96$ & $1.05 \pm 0.05$ \\
& 30 & $473 \pm 10$ & $-63.5 \pm 1.0$ & $-26.8 \pm 0.9$ & $-36.7 \pm 0.1$ & & $1.02 \pm 0.02$ \\
& 37 & $753 \pm 12$ & $-69.7 \pm 2.9$ & $-33.3 \pm 2.8$ & $-36.4 \pm 0.0$ & & $1.05 \pm 0.04$ \\
\hline
\end{tabular}



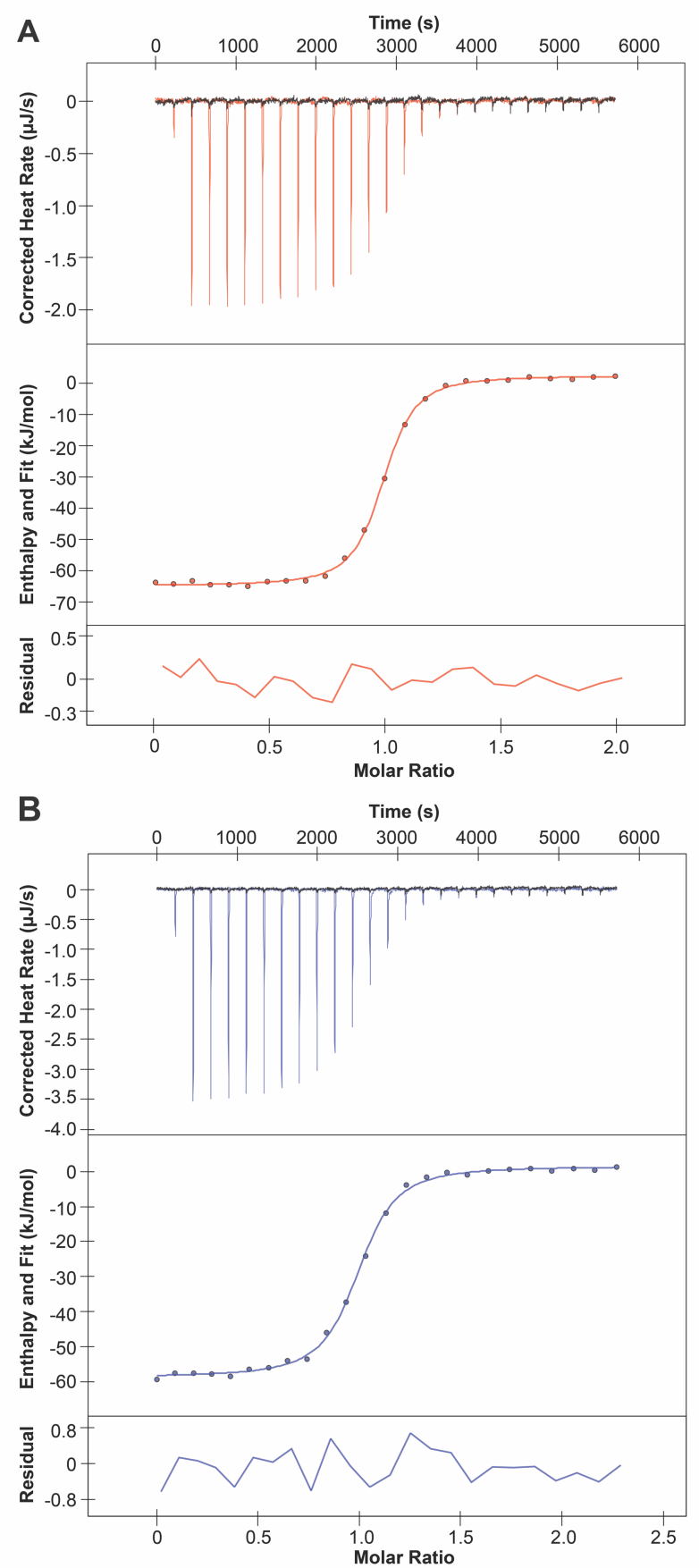

Fig. 2 Isothermal titration calorimetry (ITC) analysis of nitrate (A, red) and nitrite (B, blue) binding to $\mathrm{NrtA}$ at $20^{\circ} \mathrm{C}$. For each anion, a representative thermogram (top), isotherm (middle), and residual (bottom) plot is shown. In each thermogram, titration of the anion into the buffer alone (black) is included. All experiments were carried out in $20 \mathrm{mM} \mathrm{HEPES}$ with $50 \mathrm{mM} \mathrm{NaCl}$ at $\mathrm{pH}$ 7.7. 
We next tested the effect of temperature on nitrate and nitrite binding from 10 to $37^{\circ} \mathrm{C}$ (Tables 1 , S5; Figs. S7, S11-S13). Across this temperature range, the interaction between nitrate and NrtA is enthalpically favorable and entropically unfavorable with no significant differences in the Gibbs free energy $\left(\Delta G \approx-40 \mathrm{~kJ} \cdot \mathrm{mol}^{-1}\right)$ or corresponding thermodynamic parameters $\left(\Delta \mathrm{H} \approx-68 \mathrm{~kJ} \cdot \mathrm{mol}^{-1}\right.$, $\left.\mathrm{T} \Delta \mathrm{S} \approx-27 \mathrm{~kJ} \cdot \mathrm{mol}^{-1}\right)$. As expected, the binding affinity of nitrate for NrtA was weaker with increasing temperature and can be ranked as follows: $10{ }^{\circ} \mathrm{C}\left(K_{d}=48 \pm 8 \mathrm{nM}\right)<20{ }^{\circ} \mathrm{C}\left(K_{d}=57 \pm 23 \mathrm{nM}\right)<$ $30{ }^{\circ} \mathrm{C}\left(K_{d}=88 \pm 9 \mathrm{nM}\right)<37^{\circ} \mathrm{C}\left(K_{d}=115 \pm 8 \mathrm{nM}\right)$. Compared to nitrate, the affinity of nitrite for NrtA is weaker at each temperature and can be ranked as follows: $10{ }^{\circ} \mathrm{C}\left(K_{d}=187 \pm 16 \mathrm{nM}\right)<$ $20^{\circ} \mathrm{C}\left(K_{d}=230 \pm 33 \mathrm{nM}\right)<30^{\circ} \mathrm{C}\left(K_{d}=473 \pm 10 \mathrm{nM}\right)<37^{\circ} \mathrm{C}\left(K_{d}=753 \pm 12 \mathrm{nM}\right)$. This interaction is also enthalpically favorable and entropically unfavorable, but with a larger temperature dependent change in both thermodynamic parameters. From $10^{\circ} \mathrm{C}$ to $37^{\circ} \mathrm{C}$, the $\Delta \mathrm{H}$ for nitrite ranges from ca. -56 to $-70 \mathrm{~kJ} \cdot \mathrm{mol}^{-1}$ and $\mathrm{T} \Delta \mathrm{S}$ for nitrite ranges from ca. -20 to $-33 \mathrm{~kJ} \cdot \mathrm{mol}^{-1}$. However, the Gibbs free energy $\left(\Delta \mathrm{G} \approx-36 \mathrm{~kJ} \cdot \mathrm{mol}^{-1}\right)$ remains unchanged due to the enthalpyentropy compensation. ${ }^{36,37}$

One noteworthy observation is that the $\Delta G$ for nitrate is $4 \mathrm{~kJ} \cdot \mathrm{mol}^{-1}$ more favorable than that for nitrite. It is possible that this difference is due to the entropic contributions to binding, particularly given that the enthalpic contributions are comparable for both anions at $37^{\circ} \mathrm{C}$. This can also be seen in the heat capacity change for nitrate $\left(\Delta \mathrm{Cp}=-209 \pm 45 \mathrm{~J} \cdot \mathrm{mol}^{-1} \cdot \mathrm{K}^{-1}\right)$ and nitrite $(\Delta \mathrm{Cp}=-463$ $\left.\pm 96 \mathrm{~J} \cdot \mathrm{mol}^{-1} \cdot \mathrm{K}^{-1}\right)$. Since the $\Delta \mathrm{Cp}$ value can be linked to changes in the solvent-accessible surface area (SASA) of the protein, it is likely that NrtA undergoes a different structural rearrangement to bind each anion. ${ }^{38,39}$

To support these conjectures, we employed MD for apo, nitrate, and nitrite-bound NrtA at $37^{\circ} \mathrm{C}$ (Fig. 3, left panel; see Methods). ${ }^{40,41}$ For each system, equilibration was followed by three independent $100 \mathrm{~ns}$ MD simulations. We first monitored distances within the nitrate binding pocket as defined in Figure 1 (see also Figs. S14-S28). In apo NrtA, water molecules transiently occupied the nitrate binding site (Supporting movie 1). In the bound forms, the distances between the nitrogen atom of each anion and the alpha carbons $(\mathrm{C} \alpha)$ of the coordinating residues W102, $\mathrm{H} 196, \mathrm{G} 240$, and K269 remain unchanged throughout the trajectories. Moreover, the shortest distances between the oxygen atoms of both anions and the closest side chain atoms of the coordinating residues described above are maintained within $4 \AA$, which is in close agreement with the nitrate-bound crystal structure (Table S6). ${ }^{9}$ Surprisingly, the interactions between nitrate and nitrite with Q155 are lost because the sidechain rotates out of the binding pocket resulting in distances greater than $4 \AA$ (Figs. S16, S24). This rearrangement is stabilized by nearby residues, including N153, H196, E268, and K269 (Fig. S29). To compensate, the hydroxyl group of T190 forms hydrogen bonding interactions (within $4 \AA$ ) with both anions (Figs. S17, S25). Likely due to these rearrangements, water molecules are transiently found near the anions (Supporting movies 2, 3 for nitrate and nitrite, respectively). Together, these observations are unique to our MD simulations. In solution, it is likely that water molecules in the binding pocket of apo NrtA are displaced upon anion binding. Moreover, in the bound forms the binding pocket could be partially hydrated to enable anion sequestration or delivery to downstream proteins, NrtBCD. 

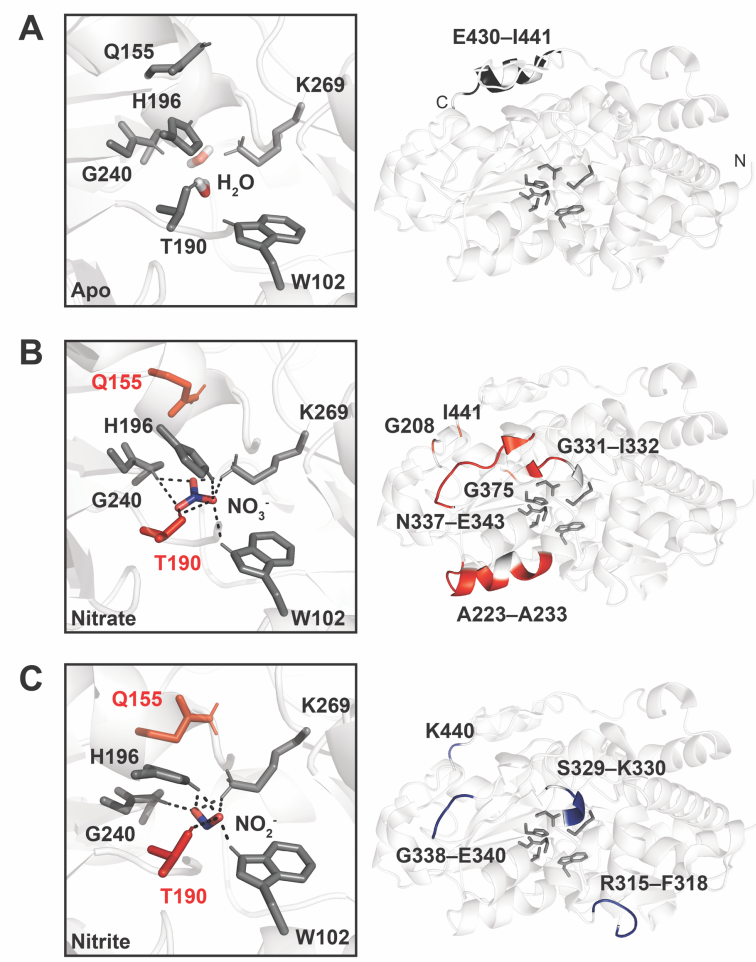

Fig. 3 Molecular dynamics (MD) simulations reveal that NrtA undergoes different conformational changes when bound to nitrate versus nitrite. Snapshots from the MD simulations (left) and residues that have C $\alpha$ RMSF values greater than $0.4 \AA$ along the PC1 direction (right) for (A) apo, (B) nitrate-bound, and (C) nitritebound NrtA. Polar residues as defined in Figure 1 are shown as sticks in gray or red with hydrogen bonding or electrostatic interactions with the anions shown as dashed lines. Water, nitrate, and nitrite molecules are shown as sticks with all oxygen atoms in red, nitrogen atoms in blue, and hydrogen atoms in white. Each residue is labeled with the single letter amino acid abbreviation and corresponding sequence number. In panel $A$ (right), the $\mathrm{N}$ - and $\mathrm{C}$-termini are labeled with the letters $\mathrm{N}$ and $\mathrm{C}$, respectively. For clarity, the same orientation is used in panels $B$ and $C$. For the panels in the right column, all residues in the colored regions are listed in Table S11.

We next investigated the global conformational changes upon anion binding from the MD trajectories using principal component analysis (PCA). ${ }^{42-44}$ Since we started with the crystal structure bound to nitrate, the root-mean-square deviation (RMSD) of the protein backbone was used to determine when the apo and nitrite-bound NrtA finished adapting to their new environments (Fig. S30). The plots of the first and second principal components (PC1 vs. PC2) reveal that nitrate and nitrite-bound NrtA adopt different conformations compared to the apo form (Fig. S31). This is also supported by the fact that the SASA of apo NrtA increases with both nitrate $\left(\triangle S A S A \approx 229 \AA^{2}\right)$ and nitrite $\left(\triangle S A S A \approx 59 \AA^{2}\right)$ (Table S7). Comparatively, the SASA for nitrite is less than nitrate $\left.\triangle S A S A \approx 169 \AA^{2}\right)$, which is in line with the $\triangle C p$ values described above.

To identify the residues that contribute to these differences, root mean square fluctuations (RMSF) of the $\mathrm{C} \alpha$ atoms in the apo, nitrate, and nitrite-bound forms along the PC1 direction were plotted for the three independent trajectories (Fig. S32). To draw comparisons between all the 
datasets, only the residues that had $\mathrm{C} \alpha \mathrm{RMSF}$ values greater than $0.4 \AA$ and were common to all trajectories were selected as shown (Fig. 3, right panel; Tables S8-S11). For the apo form, we observed conformational flexibility in the C-terminal region (E430-N431, Q433-A434, L436D437, L439, I441); however, this same region is rigidified upon anion binding. This is in line with previous reports that speculate changes in the $\mathrm{C}$-terminal region may facilitate anion binding and transport. $^{9}$ Interestingly, the helix (A223-Q224, A227-N228, K230-A233) extending from the binding pocket residue P222 is coupled to nitrate but not to nitrite binding. Outside the binding pocket, isolated residues and flexible loops are also involved. For the nitrate-bound form, these include G208, G331-1332, N337-E343, G375, and 1441; for the nitrite-bound form, these include R315-F318, S329-K330, G338-E340, and K440. Taken together, our data suggests that the residues within the binding pocket are selectively coupled to residues outside the binding pocket, enabling NrtA to distinguish between nitrate and nitrite.

To summarize, we have presented the biophysical and in silico characterization of NrtA from Synechocystis sp. PCC 6803 as a protein-based host for nitrate and nitrite. Thermodynamic analysis shows that NrtA recognizes nitrate with greater affinity than nitrite, with a $4 \mathrm{~kJ} \cdot \mathrm{mol}^{-1}$ difference in the Gibbs free energy. Even though the binding is enthalpically favorable in both cases, it is plausible that differences between the two anions could arise from entropic contributions to binding and/or changes in the SASA of the protein. These conclusions are supported by our MD simulations that reveal how residues within the binding pocket rearrange in a similar fashion, while residues outside the binding pocket differentially reorganize to accommodate each anion. Through our hybrid approach, we have begun to elucidate how NrtA can recognize anions with different physical properties in its biological context with extensions to general principles of anion binding proteins. Looking forward, we anticipate that the knowledge gained from our investigations with NrtA and other biological supramolecular hosts could more broadly guide and enable the design of synthetic supramolecular hosts for aqueous anion recognition.

\section{Acknowledgments}

The authors thank Dr. Gabriele Meloni, Dr. John Sibert, and members of the Dodani Lab for helpful discussions. The authors also thank Dr. Chad A. Brautigam and Dr. Shih-Chia Tso (Macromolecular Biophysics Resource at UT Southwestern), Dr. Yeun Hee Kim (Genome Center at UT Dallas), and Dr. Christopher Simmons (Office of Information Technology at UT Dallas) for expert technical assistance. The authors acknowledge the Texas Advanced Computing Center (TACC) at UT Austin for providing HPC resources (http://www.tacc.utexas.edu). S.C.D acknowledges support from UT Dallas, the Welch Foundation (AT-1918-20170325, AT-206020210327), and the National Institute of General Medical Sciences of the National Institutes of Health (R35GM128923). This work is the sole responsibility of the authors and does not represent the views of the funding sources.

\section{Conflicts of interest}

There are no conflicts to declare. 


\section{References}

1. X. Zhang, B. B. Ward and D. M. Sigman, Chem. Rev., 2020, 120, 5308-5351.

2. C. Moreno-Vivián and E. Flores, in Biology of the Nitrogen Cycle, Elsevier, 2007, pp. 263282.

3. Y.-Y. Wang, Y.-H. Cheng, K.-E. Chen and Y.-F. Tsay, Annu. Rev. Plant Biol., 2018, 69, 85-122.

4. A. W. DeMartino, D. B. Kim-Shapiro, R. P. Patel and M. T. Gladwin, Br. J. Pharmacol., 2019, 176, 228-245.

5. E. A. Vidal, J. M. Alvarez, V. Araus, E. Riveras, M. D. Brooks, G. Krouk, S. Ruffel, L. Lejay, N. M. Crawford, G. M. Coruzzi and R. A. Gutiérrez, Plant Cell, 2020, 32, 2094-2119.

6. M. Karwowska and A. Kononiuk, Antioxidants, 2020, 9, 241.

7. A. Romanelli, D. X. Soto, I. Matiatos, D. E. Martínez and S. Esquius, Sci. Total Environ., 2020, 715, 136909.

8. X. Zhang, T. Zou, L. Lassaletta, N. D. Mueller, F. N. Tubiello, M. D. Lisk, C. Lu, R. T. Conant, C. D. Dorich, J. Gerber, H. Tian, T. Bruulsema, T. M. Maaz, K. Nishina, B. L. Bodirsky, A. Popp, L. Bouwman, A. Beusen, J. Chang, P. Havlík, D. Leclère, J. G. Canadell, R. B. Jackson, P. Heffer, N. Wanner, W. Zhang and E. A. Davidson, Nat. Food, 2021, 2, 529-540.

9. N. M. Koropatkin, H. B. Pakrasi and T. J. Smith, Proc. Natl. Acad. Sci. U.S.A., 2006, 103, 9820-9825.

10. H. Yan, W. Huang, C. Yan, X. Gong, S. Jiang, Y. Zhao, J. Wang and Y. Shi, Cell Rep., 2013, 3, 716-723.

11. V. Niemann, M. Koch-Singenstreu, A. Neu, S. Nilkens, F. Götz, G. Unden and T. Stehle, J. Mol. Biol., 2014, 426, 1539-1553.

12. J. L. Parker and S. Newstead, Nature, 2014, 507, 68-72.

13. C. Coelho and M. J. Romão, Protein Sci., 2015, 24, 1901-1911.

14. J. M. Fox, K. Kang, W. Sherman, A. Héroux, G. M. Sastry, M. Baghbanzadeh, M. R. Lockett and G. M. Whitesides, J. Am. Chem. Soc., 2015, 137, 3859-3866.

15. S. Horrell, D. Kekilli, R. W. Strange and M. A. Hough, Metallomics, 2017, 9, 1470-1482.

16. D. Martín-Mora, Á. Ortega, M. A. Matilla, S. Martínez-Rodríguez, J. A. Gavira and T. Krell, MBio, 2019, 10, e02334-18.

17. W. Yao, K. Wang, A. Wu, W. F. Reed and B. C. Gibb, Chem. Sci., 2021, 12, 320-330.

18. Y. Marcus, Biophys. Chem., 1994, 51, 111-127.

19. O. A. Okunola, P. V. Santacroce and J. T. Davis, Supramol. Chem., 2008, 20, 169-190.

20. R. Dutta and P. Ghosh, Chem. Commun., 2015, 51, 9070-9084.

21. Q. He, Z. Zhang, J. T. Brewster, V. M. Lynch, S. K. Kim and J. L. Sessler, J. Am. Chem. Soc., 2016, 138, 9779-9782.

22. S. S. R. Namashivaya, A. S. Oshchepkov, H. Ding, S. Förster, V. N. Khrustalev and E. A. Kataev, Org. Lett., 2019, 21, 8746-8750.

23. I. A. Rather, S. A. Wagay and R. Ali, Coord. Chem. Rev., 2020, 415, 213327.

24. J. N. Tutol, W. Peng and S. C. Dodani, Biochemistry, 2019, 58, 31-35.

25. J. N. Tutol, H. C. Kam and S. C. Dodani, ChemBioChem, 2019, 20, 1759-1765.

26. J. N. Tutol, J. Lee, H. Chi, F. N. Faizuddin, S. S. Abeyrathna, Q. Zhou, F. Morcos, G. Meloni and S. C. Dodani, Chem. Sci., 2021, 12, 5655-5663.

27. T. Omata, X. Andriesse and A. Hirano, Mol. Gen. Genet. MGG, 1993, 236, 193-202.

28. Y. Ohashi, W. Shi, N. Takatani, M. Aichi, S. Maeda, S. Watanabe, H. Yoshikawa and T. Omata, J. Exp. Bot., 2011, 62, 1411-1424.

29. S. Maeda and T. Omata, J. Biol. Chem., 1997, 272, 3036-3041.

30. D. Nagore, B. Sanz, J. Soria, M. Llarena, M. J. Llama, J. J. Calvete and J. L. Serra, Biochim. Biophys. Acta, 2006, 1760, 172-181. 
31. G. A. Holdgate and W. H. J. Ward, Drug Discov. Today, 2005, 10, 1543-1550.

32. F. H. Niesen, H. Berglund and M. Vedadi, Nat. Protoc., 2007, 2, 2212-2221.

33. N. M. Koropatkin, D. W. Koppenaal, H. B. Pakrasi and T. J. Smith, J. Biol. Chem., 2007, 282, 2606-2614.

34. R. Perozzo, G. Folkers and L. Scapozza, J. Recept. Signal Transduct., 2004, 24, 1-52.

35. R. N. Goldberg, N. Kishore and R. M. Lennen, J. Phys. Chem. Ref. Data, 2002, 31, 231370.

36. J. D. Chodera and D. L. Mobley, Annu. Rev. Biophys., 2013, 42, 121-142.

37. J. M. Fox, M. Zhao, M. J. Fink, K. Kang and G. M. Whitesides, Annu. Rev. Biophys., 2018, 47, 223-250.

38. N. V. Prabhu and K. A. Sharp, Annu. Rev. Phys. Chem., 2005, 56, 521-548.

39. S. Vega, O. Abian and A. Velazquez-Campoy, Biochim. Biophys. Acta, 2016, 1860, 868878.

40. W. Humphrey, A. Dalke and K. Schulten, J. Mol. Graph., 1996, 14, 33-38.

41. J. C. Phillips, D. J. Hardy, J. D. C. Maia, J. E. Stone, J. V. Ribeiro, R. C. Bernardi, R. Buch, G. Fiorin, J. Hénin, W. Jiang, R. McGreevy, M. C. R. Melo, B. K. Radak, R. D. Skeel, A. Singharoy, Y. Wang, B. Roux, A. Aksimentiev, Z. Luthey-Schulten, L. V. Kalé, K. Schulten, C. Chipot and E. Tajkhorshid, J. Chem. Phys., 2020, 153, 044130.

42. N. Michaud-Agrawal, E. J. Denning, T. B. Woolf and O. Beckstein, J. Comput. Chem., 2011, 32, 2319-2327.

43. C. C. David and D. J. Jacobs, in Methods Mol. Biol., Humana Press, NJ, 2014, vol. 1084, pp. 193-226.

44. R. Gowers, M. Linke, J. Barnoud, T. Reddy, M. Melo, S. Seyler, J. Domański, D. Dotson, S. Buchoux, I. Kenney and O. Beckstein, in Proceedings of the 15th Python in Science Conference, 2016, 98-105. 